\title{
New Millennium: New Expectations for Alzheimer's Disease?
}

\author{
Jean-Marc Orgogozo
}

A major goal in the pharmacological treatment of Alzheimer's disease (AD) is to produce a clinically relevant benefit that is sustained in the long term. In order to meet this objective we have to ask two questions: How do we measure benefit, and what strategies can we use to prolong efficacy? To address these and other important issues in the management of $\mathrm{AD}$, a panel of international experts gave presentations at a symposium, 'The Modern Treatment of Alzheimer's Disease', which was held at the congress of the European Federation of Neurological Society (EFNS), in Lisbon, in September 1999. The articles that follow are based on these presentations.

The central feature of $\mathrm{AD}$ is a progressive deterioration in a person's cognitive functions, particularly in memory. The assessment of cognitive function is therefore essential in judging treatment outcome, as discussed by Professor Winblad in this supplement. To date, the cognitive subscale of the Alzheimer's Disease Assessment Scale, ADAS-cog, which assesses memory, attention, orientation and language, is an internationally accepted standard measure of cognitive function in $\mathrm{AD}$ drug trials. In 6month studies, an improvement relative to placebo of at least 3-4 points on ADAS-cog is judged to be clinically relevant. Untreated patients with $\mathrm{AD}$ would be expected to deteriorate by 5-11 points per year. Therefore, in 12month studies, maintaining cognitive function at or near baseline values is considered to be a clinically significant outcome. However, this result in itself is not sufficient, as

\begin{tabular}{ll}
\hline KARGER & ( ) 2000 S. Karger AG, Basel \\
1420-8008/00/0117-0001\$17.50/0 \\
$\begin{array}{l}\text { Fax +4161306 12 34 } \\
\begin{array}{l}\text { E-Mail karger@karger.ch } \\
\text { www.karger.com }\end{array}\end{array}$ & $\begin{array}{l}\text { Accessible online at: } \\
\text { www.karger.com/journals/dem }\end{array}$
\end{tabular}

other dimensions in AD symptomatology are also very important for both the patient and the caregivers, namely the ability to perform activities of daily living (ADL) and patients' global functioning, in which behavioural disturbances play an important role.

Professor Winblad emphasized that treatment success should not be judged purely on cognitive function. AD is a devastating illness whose impact can and should be assessed beyond changes on a psychometric scale. As occurs with memory and other cognitive skills, there is a progressive decline in a patient's ability to carry out first the more complex and then even basic activities of daily living. Once the ability to perform a daily activity is lost because of $\mathrm{AD}$, it is rarely recovered. This has considerable implications for the patient, the carer and healthcare resources. A treatment that can preserve the patients' functional ability will prolong their independence, and therefore reduce the burden on the caregiver. There are several contributing factors to caregiver burden, not least of which is time spent by the caregiver supervising and assisting a patient. Caregiver time, together with the use of hospital and physician services, forms a large part of resource utilization, which accounts for most of the economic cost of AD. Professor Winblad also highlighted other key aspects of AD such as behavioural and psychological symptoms, which can cause considerable distress to caregivers.

As our understanding of the biochemical and pathological disturbances of $\mathrm{AD}$ grows, so does the number of dif-

Jean-Marc Orgogozo

CHU Pellegrin, University of Bordeaux

F-33076 Bordeaux Cedex (France)

Tel. +33 55679 6004, Fax +3355698 1378

E-Mail j.m.orgogozo@neuro.u-bordeaux2.fr 
ferent potential pharmacological approaches. However, the enhancement of cholinergic function through acetylcholinesterase (AChE) inhibition remains the most successful approach so far for treating AD. By increasing the availability of acetylcholine (ACh) in the synaptic cleft, AChE inhibitors go some way to correcting the cholinergic deficits that occur in AD. Professor Maelicke, however, points out that there are now plenty of data to suggest that enhancing nicotinic-receptor function is a promising treatment strategy for AD. Professor Maelicke described a novel mechanism, i.e. allosteric modulation of nicotinic receptors, which increases the sensitivity of nicotinic receptors to ACh. This mechanism allows nicotinic receptors to make a full response to ACh even when there are reduced levels of ACh available. Presynaptic nicotinic receptors control the release of several neurotransmitters, including ACh and glutamate, which are deficient in AD. Therefore, by potentiating nicotinic receptor function, allosteric modulation could correct both the cholinergic and non-cholinergic deficits of AD. Professor Maelicke reviewed the evidence that modulation of nicotinic receptors could alleviate the cognitive and non-cognitive symptoms of AD. In contrast to full agonists, allosteric modulators are unlikely to desensitize nicotinic receptors and therefore may prolong clinical benefit.

Dr. Lilienfeld and Professor Blesa reviewed the cognitive and non-cognitive benefits of galantamine, a novel agent for the treatment of AD. Galantamine has a dual mechanism of action on the cholinergic system, combining allosteric modulation of nicotinic receptors with AChE inhibition. Galantamine produces a significantly better outcome on ADAS-cog than placebo and maintains patients' cognitive function above baseline in 6-month, placebo-controlled studies. The superiority of galantamine over placebo increases during the 6-month study peri- od. This sustained efficacy has been confirmed in a 12month study. Patients were initially randomized to galantamine $(24 \mathrm{mg} /$ day or $32 \mathrm{mg} /$ day $)$ or placebo. After 6 months, all patients who continued in the study received galantamine $24 \mathrm{mg} / \mathrm{day}$. The group of patients who received galantamine $24 \mathrm{mg}$ /day for 12 months maintained their cognitive function at baseline level. This indicates that galantamine produces clinically significant benefit for at least 12 months. The group of patients who received placebo for the first 6 months followed by galantamine for 6 months experienced a modest but definite decline in cognitive function at the end of 12 months, suggesting that early treatment with galantamine produces a better long-term outcome than later treatment. The clinical relevance of galantamine's effects on cognitive function is also supported by its impact on patients' daily functioning. At the end of the 12-month study, the ability to carry out ADL was better preserved in galantamine-treated patients than in those patients who initially received placebo.

Data from a 5-month placebo-controlled study also suggested that galantamine postpones the emergence of behavioural symptoms. The favourable effects of galantamine on patients' functioning and behavioural symptoms alleviated caregiver burden, as measured by caregiver time. These data suggest that combined cholinergic mechanisms may produce additional clinical benefits for patients with $\mathrm{AD}$ and their carers.

Alzheimer's disease has been described as 'a funeral that never ends'. The use of a broad range of outcome measures to judge the benefits of potential treatments, and the development of novel drugs, like galantamine, will hopefully reduce the burden of this devastating illness. 